\title{
Social network and high performance in smart communications
}

\author{
Jongsung Kim • Philip S. Yu • Nasrullah Memon
}

Published online: 28 September 2013

(C) Springer Science+Business Media New York 2013

Recently, social computing and networking has been driving dramatic evolution in the way people communicate and interact with each other, attracting great attention from the research community as well as the business world. Social networks not only include Web 2.0 elements such as blogs, twitter, and wikis, etc., but also stand for a collection of the technologies that gather, process, compute, and visualize social information and the studies that model and analyze dynamics of participants in social networks. With the advent of the social computing era, this special issue aims at reporting the most recent progresses, trends, and concerns in social network and high performance in smart communications.

This special issue provides a forum for participants from the computing and social science communities and publishes state-of-art research papers. Furthermore, we expect that the special issue and its publications will trigger related research and technology innovation in this important subject. This special issue is aimed to solicit research results on the following topics of interest, but not limited:

- Social Network System Design and Architectures.

\footnotetext{
J. Kim (凶)

Department of Mathematics, Kookmin University, Kookmin University, 77 Jeongneung-Ro, Seongbuk-Gu, Seoul 136-702, Republic of Korea

e-mail: jongsung.k@gmail.com

P.S. Yu

Department of Computer Science, University of Illinois at Chicago, 851 S. Morgan St., Rm 1138 SEO, Chicago, IL 60607, USA

e-mail: psyu@cs.uic.edu

N. Memon

The Maersk Mc-Kinney Moller Institute, University of Southern Denmark, Campusvej 55, 5230 Odense, Denmark

e-mail: memon@mmmi.sdu.dk
} 
- Peer-to-Peer System for Social Networks.

- Advanced Social Computing Technologies.

- Collaborative and Multiagent Systems for Social Computing.

- Privacy, Trust, and Security in Social Networks.

- Supercomputing based Social Networks.

- High Performance in Smart Communications.

- Social Network and Smart-phone Services.

- Semantic Web Technologies and Applications for Social Network.

- Context-Awareness and Context Sharing for Social Network.

- Social Media Business Models.

- Location Aware Services for Social Network.

- E-Learning, Edutainment, and Infotainment in Social Network.

- Video Distribution (IPTV, VoD) in Social Network.

- Web Page Ranking Informed by Social Media.

- Social and Ethical Issues of Networked World.

- Data Mining and Machine Learning for Social Networks.

- Social Behavior Modeling.

- Social Network Services and Case Studies.

We received many manuscripts: Each manuscript was blindly reviewed by at least three reviewers consisting of guest editors and external reviewers. After a rigorous review process, nine manuscripts were finally selected for this Special Issue. The selected manuscripts are as follows:

1. The QoS-based MCDM system for SaaS ERP applications with Social Network, Jong Huk Park, Hwa-Young Jeong.

2. Dynamic FOAF management method for social networks in the social web environment, Jong-Soo Sohn, In-Jeong Chung.

3. An energy-efficient clustered distributed coding for large-scale wireless sensor networks, Yuexing Peng, Yonghui Li, Lei Shu, Wenbo Wang.

4. A uplink radio resource allocation scheme for localized SC-FDMA transmission in LTE network, Yen Wen Chen, Shaoh Chen Ke.

5. A disk bandwidth allocation mechanism with priority, Xibin Wang, Xia Xie, Hai Jin, Xuanhua Shi, Wenzhi Cao, Xijiang Ke.

6. Churn-aware optimal layer scheduling scheme for scalable video distribution in super-peer overlay networks, Yong-Hyuk Moon.

7. Efficient algorithms for team formation with a leader in social networks, MingChin Juang, Chen-Che Huang, Jiun-Long Huang.

8. MODM: multiobjective diffusion model for dynamic social networks using evolutionary algorithm, Iram Fatima, Muhammad Fahim, Young-Koo Lee, Sungyoung Lee.

9. Analysis and effects of smart home dataset characteristics for daily life activity recognition, Iram Fatima, Muhammad Fahim, Young-Koo Lee, Sungyoung Lee.

Finally, we would like to thank all authors for their contributions to this special issue. We also extend our thanks to the following external reviewers for their excellent job in reviewing the manuscripts: Bok-Kyu Joo, Chih-Lin Hu, Dilip Kumar, Greg Eisenhauer, Guan Donghai, Guojun Wanga, Hye-Jung Jung, I-Hsuan Peng, Jeangun 
Kang, Jehad Sarkar, Junhui Zhao, Ju Yeon Jo, Kesavath Vasudevan, Kun-Ta Chuang, Li-Der Chou, Manuel Cebrian, Minglu Li, P. Suresh, Phillip M. Dickens, Rehan Akbar, Salaja Silas, Seong Hwan Kim, TAN Zhen-Hua, Tim Bell, Tran Minh, Yong-Moo Suh, Yuan Weiwei, Yuseop Lee, and Zhi Ning Chen. 